\title{
Emerging market equity benchmarks for Japanese investors: countries, sectors or styles?
}

\author{
Harsh Parikh ${ }^{1}$ \\ Published online: 25 June 2019 \\ (c) The Author(s) 2019
}

\begin{abstract}
Japanese investors maybe considering adding emerging market (EM) equities to their portfolios. What type of baseline EM exposure might be most suitable for Japanese investors? Given recent improvements in benchmark technology, more extensive data coverage and empirical research in the underlying drivers of equity returns, Japanese investors can consider selecting, or designing, an EM benchmark that is most advantageous. The author shows that a traditional market-capitalization EM benchmark (e.g., MSCI EM index) may not be best-suited. Based on recent research showing that EM returns are influenced by sector and style exposures, in addition to country exposures, the author presents three alternative EM benchmarks that have provided better diversification, risk-adjusted returns and lower performance drawdowns for Japanese investors, compared to a traditional EM benchmark. Japanese investors should consider adopting one of these alternative EM benchmarks to represent their baseline EM allocation.
\end{abstract}

Keywords Emerging market equities $\cdot$ Equity benchmarks $\cdot$ Non-cap-weighted indexes

\section{Introduction}

Emerging market (EM) equities currently comprise $11 \%$ of free float, all-country market capitalization, so it is no surprise that many investors include EM as part of their longterm asset allocation. ${ }^{1}$ This EM allocation has performed well. Over the last 15 years, although realized risk $^{2}$ in EM was higher than developed market (DM) equities, EM performed better, even on a risk-adjusted basis. An allocation to EM may continue to have return and diversification benefits going forward.

Japanese investors may be considering adding EM to their portfolios. ${ }^{3,4}$ What form should this EM allocation take? Adding a long-term asset allocation to an asset class involves selecting a benchmark to represent this exposure. A benchmark serves not only to represent the asset class'

Electronic supplementary material The online version of this article (https://doi.org/10.1057/s41260-019-00123-7) contains supplementary material, which is available to authorized users.

Harsh Parikh

harsh.parikh@pgim.com

1 PGIM, Newark, NJ, USA risk and return properties but also represents the investor's baseline exposure. Investors can then choose either passive exposure to that benchmark or employ an active asset manager. Given recent improvements in benchmark technology, more extensive data coverage and empirical research in the underlying drivers of equity returns, Japanese investors can select, or design, an EM benchmark that is customized for their needs.

\footnotetext{
1 Source: MSCI. MSCI has not approved, reviewed or produced this report, makes no express or implied warranties or representations and is not liable whatsoever for any data in the report. You may not redistribute the MSCI data or use it as a basis for other indices or investment products.

${ }^{2}$ No risk management technique can guarantee the mitigation or elimination of risk in any market environment.

3 Elanor Warnock, "Japan's Public Servants Turn to Emerging Markets," The Wall Street Journal, September 11, 2013. The author writes that the Pension Fund Association for Local Government Officials (PAL), a \$170bn reserve fund started to invest in EM equities.

4 Madison Marriage, "Japanese pension funds leap into foreign and local equities," Financial Times, May 31, 2015. The author writes that many public pension funds which never invested in equities are expected to adopt policies similar to Japan's Government Pension Investment Fund and increase their exposure to domestic and foreign stocks. This article may help such pension investors to select an EM benchmark.
} 
How might a Japanese invest or select, or design, an EM benchmark? An obvious choice is a traditional market-capitalization-weighted EM index (e.g., the MSCI EM index comprising large- and mid-capitalization stocks). This type of index uses stocks' market-capitalization weights both to combine stocks by country and then to combine countries. All eligible EM countries are included in the benchmark.

However, the traditional EM benchmark has exposures that may not be ideal for Japanese investors. For example, the MSCI EM index has more than 50\% of its market value represented by just three countries: China, Korea and Taiwan. ${ }^{5}$ After the USA, these three neighboring countries are the largest export destinations for Japan. In addition, almost $50 \%$ of the traditional EM benchmark is represented by exposure to just two sectors: Financials and Technology. These two sectors are also heavily represented in developed equity markets, with more than a $30 \%$ combined weight. Are these EM country and sector concentrations desirable for Japanese investors? After all, the decision to add EM exposure is motivated by a desire for additional portfolio diversification, as well as higher returns. ${ }^{6}$

We examine several alternative EM benchmarks from a Japanese investor's viewpoint. These alternatives are based both on recent research identifying stock attributes that better explain their returns and risk and on portfolio construction techniques that may provide better diversification and risk-adjusted returns. As mentioned, a traditional EM benchmark uses market-capitalization weights to both group stocks by country and then to group countries. Grouping EM stocks by country may seem reasonable if a stock's country exposure is the major attribute that drives its returns and the variation of returns across stocks. A benchmark defined in terms of "countries" then helps investors identify their sources of potential returns and risk in terms of their country exposures. A Japanese investor would then be concerned with how these country exposures interact with the rest of their portfolio.

However, research shows that a stock's "sector" (i.e., industry) and "style" (such as value, momentum, size, etc.)

\footnotetext{
${ }^{5}$ Such EM country concentration may not be ideal say for an institutional US investor either. Our proposed alternative EM benchmarks may be adopted by the US investor.

${ }^{6}$ Refer to Wilcox (1994), author discusses country concentration in EAFE and recommended either using equal-weighted or minimum variance portfolio using country aggregates and accounting for the investor's whole portfolio. For the author, EM was beyond the scope. While the author only considered country aggregates, in our analysis, we also evaluate alternative EM benchmarks based on sector and style aggregates, as sectors and styles are also important drivers of equity returns. Recently, a lot more focus is given to benchmark construction using style aggregates but because countries and sectors are also drivers of equity returns benchmarks constructed using all three aggregates should be compared.
}

exposures are also important attributes that drive its risk, returns and the variation of returns across stocks. ${ }^{7}$ While return dispersion across stocks due to country exposure has usually been higher than due to sector or style exposure, in 2010-2012, all three groups were equally responsible for dispersion in returns (see Exhibit 1). In addition, dispersion due to country exposure has been declining relative to dispersion due to sector or style exposure. This suggests that EM investors might be better served with a benchmark that focuses less on country groupings and more on sector or style groupings. Japanese investors might reasonably be concerned with how these sector or style exposures interact with the rest of their portfolio.

Given a desired exposure metric (e.g., country, sector or style), how are these exposures to be combined into a benchmark? A traditional EM benchmark uses market-capitalization weights. However, investors can choose alternative weighting schemes that might provide better risk and return properties that better fit the investor's overall portfolio. We consider two alternative weighting schemes: equal risk contribution ("ERC") weights and equal weighting ("EW"). There are many other potential exposure weighting schemes, and some may work better than others depending on the economic regime. However, we choose to examine ERC and EW due to their long history and their focus on diversification, which may be desirable for investors in a new and volatile asset class. ${ }^{8}$

The key question we wish to address is whether a Japanese investor might be better served by an alternative EM benchmark compared to the traditional MSCI EM benchmark. ${ }^{9}$ Specifically, we consider alternative benchmarks based on country, sector or style exposures, which are combined either by equal risk contribution or equal weights. We show how the alternative benchmarks have performed in various economic environments both as a stand-alone investment and in a portfolio as a substitute for a portion of the Japanese investor's existing DM ex-Japan equity allocation.

Compared to the traditional market-capitalizationweighted EM index, the alternative EM benchmarks, using

\footnotetext{
${ }^{7}$ See "Drivers of Returns in Emerging Markets Equities: The Growing Importance of Stock Selection", QMA, January 2014.

${ }^{8} \mathrm{EW}$ and ERC portfolios have been shown to be efficient out-ofsample. If all assets have equal mean, variance correlation coefficient than the EW portfolio would be the tangency portfolio. If all assets have equal Sharpe ratio and correlations than the ERC portfolio would be the tangency portfolio. Refer to Maillard et al. (2010). Therefore, ex-ante EW and ERC portfolios are good proxy for a tangency portfolio. In contrast, cap-weighted portfolios are not welldiversified and so lead to an inefficient portfolio. Refer to Amenc et al. (2012).

9 All three alternative benchmarks could be constructed and published by index providers.
} 


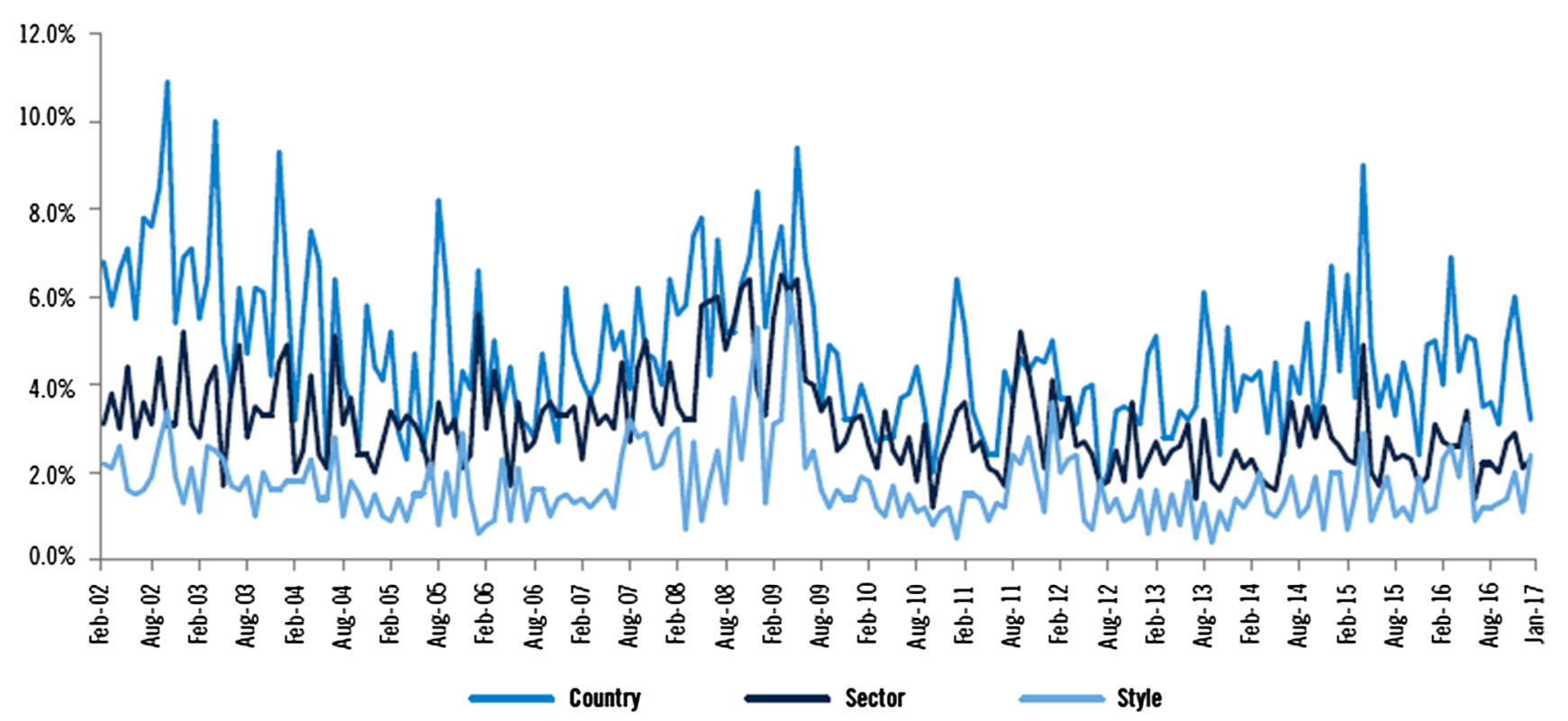

Exhibit 1 Country, sector and style return dispersion (February 2002-January 2017). Note: We define monthly return dispersion as the cross-sectional standard deviation of returns (in JPY, unhedged) of the group's constituents each month. Country group dispersion is measured using 11 cap-weighted country portfolios' returns; sec-

equal risk contribution, appear to be better-suited for Japanese investors. Among the three alternative benchmarks using ERC, we find that the country-based and style-based alternative EM benchmarks, on a risk-adjusted basis, have performed better than the sector-based alternative EM benchmark. If achieving lower volatility is the only investment objective, then the sector-based alternative EM benchmark does better.

\section{Role of EM in Japanese investor portfolios}

We first examine the case for adding EM exposure to a Japanese portfolio. For our analysis, we assume ${ }^{10}$ a sample Japanese portfolio allocation represented by a $50 \%$ DM equity allocation (25\% in domestic (i.e., Japanese) equities and 25\% in DM ex-Japan equities) and a 50\% bond allocation (35\% in Japanese Government Bonds (JGBs) and 15\% in global government bonds ex-Japan). ${ }^{11}$ For the moment, we assume EM is represented by the MSCI EM index. We assume all foreign equity and bond allocation in the Japanese portfolio is unhedged.

\footnotetext{
${ }^{10}$ If any assumptions used herein change, the results of our analysis may vary.

11 We proxy the stock allocation with MSCI equity indexes and bond allocation with J. P. Morgan bond indexes.
}

tor group dispersion uses 11 cap-weighted sector portfolios' returns; style group dispersion uses seven cap-weighted style portfolios' returns. Chart is provided for illustrative purposes only. Source: PGIM IAS, Datastream, MSCI, and S\&P Capital IQ

Exhibit 2 shows performance metrics for DM, DM exJapan, Japan and EM equities and global government bonds ex-Japan and JGBs from February 2002 to January 2017.

What happens to the risk and return characteristics of a Japanese investor's portfolio as the EM allocation increases from $0 \%$ ? To answer, we must specify which existing portfolio allocation is reduced to make room for the new EM allocation. This is an important issue, as a reduction in Japanese equities and/or JGBs and an increase in EM means that the Japanese investor is also increasing foreign currency exposure. To keep the allocation to yen and equities unchanged, we assume EM allocation is made by reducing allocation to DM ex-Japan. Exhibit 3a, b shows, historically, how the overall portfolio's performance would have changed as the allocation to EM was increased.

Overall, we see that a Japanese investor could have benefitted from an allocation to EM equities.

The appeal of a traditional market-capitalization-weighted index is that it reflects the investable market, so index weights are commensurate with their market weights. In addition, since the larger countries and stocks tend to have lower transaction costs, gaining exposure to a market-capitalization-weighted index may likely better control cost. Another advantage is that index weights move automatically with relative market performance, helping to keep turnover low.

However, a cap-weighted EM index has drawbacks. For example, as mentioned above, the traditional EM index has significant country and sector concentrations. Exhibit 4 shows 
Exhibit 2 Asset class-performance metrics (unhedged JPY returns; February 2002-January 2017). Source: PGIM IAS, Datastream, MSCI, and S\&P Capital IQ

\begin{tabular}{|c|c|c|c|c|c|c|}
\hline & Cap-weighte & & & & & \\
\hline & DM equities & $\begin{array}{l}\text { DM ex-Japan } \\
\text { equities }\end{array}$ & Japan equities & EM equities & $\begin{array}{l}\text { Global Govt. ex- } \\
\text { Japan bonds }\end{array}$ & Japan Govt. bonds \\
\hline Returns (annualized) & $5.6 \%$ & $5.7 \%$ & $4.5 \%$ & $8.7 \%$ & $4.6 \%$ & $2.0 \%$ \\
\hline Risk (annualized) & $19.0 \%$ & $19.6 \%$ & $18.4 \%$ & $24.1 \%$ & $9.5 \%$ & $2.1 \%$ \\
\hline Sharpe ratio & 0.28 & 0.28 & 0.23 & 0.35 & 0.47 & 0.87 \\
\hline Correl to Japan & 0.78 & 0.75 & 1.00 & 0.72 & 0.50 & -0.37 \\
\hline Correl to DM & 1.00 & 1.00 & 0.78 & 0.88 & 0.63 & -0.26 \\
\hline Correl to EM & 0.88 & 0.87 & 0.72 & 1.00 & 0.54 & -0.25 \\
\hline Max. drawdown & $-61.2 \%$ & $-61.7 \%$ & $-57.3 \%$ & $-68.2 \%$ & $-22.5 \%$ & $-4.5 \%$ \\
\hline
\end{tabular}

We proxy DM equities with MSCI World Total Return (TR) Index, DM ex-Japan equities with MSCI World ex-Japan TR index. Japan equities with MSCI Japan TR index, and EM equities with MSCI EM index. We proxy Global Govt. ex-Japan Bonds with J. P. Morgan Global Government Bond ex-Japan Index, and Japan Govt. Bonds (JGBs) with J. P. Morgan Japan Government Bond Index. Past performance is not a guarantee or a reliable indicator of future results. Chart is provided for illustrative purposes only

(a)

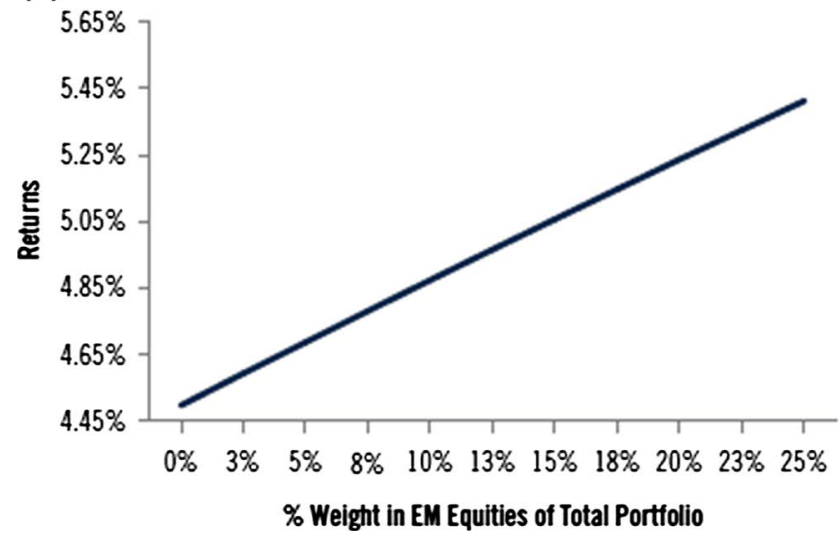

Exhibit 3 a Returns-allocation to EM equities (unheded JPY returns; February 2002-January 2017). b Sharpe ratio-allocation to EM equities (unhedged JPY returns; February 2002-January 2017).

that for the MSCI EM index, more than $50 \%$ of market value is allotted to just three of the 23 EM countries: China, Korea and Taiwan, of which China has the largest weight of $26.8 \%$ (as of 1/31/2017). However, China, Korea and Taiwan are the three largest export destinations for Japan outside of the USA. An economic shock in one or more of these countries would likely have negative reverberations in the domestic Japanese equity market, reducing the potential portfolio diversification benefit of EM exposure if using a traditional EM benchmark.

\section{Alternative EM benchmarks for Japanese investors}

If not a traditional market-cap EM benchmark, what type of benchmark might be more suitable for Japanese investors? Given advances in benchmark technology, an investor (b)

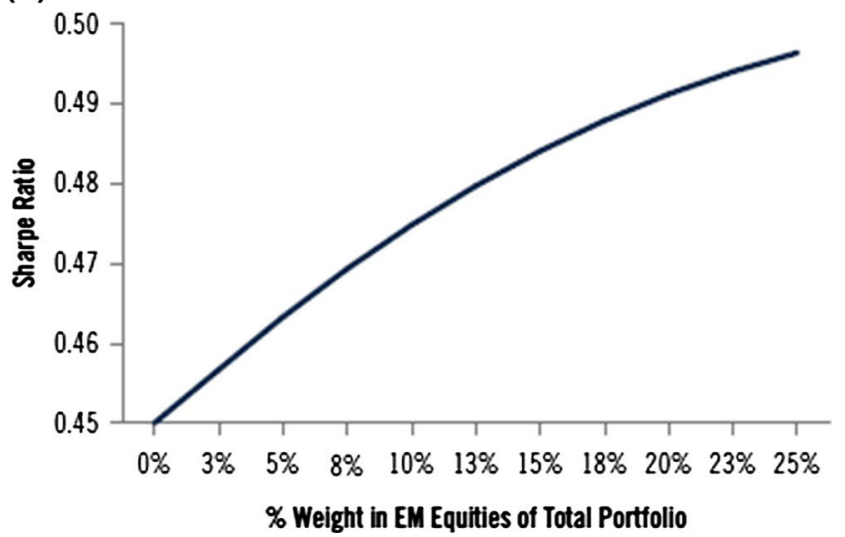

Note: Past performance is not a guarantee or a reliable indicator of future results. Chart is provided for illustrative purposes. Source: PGIM IAS, Datastream, MSCI, and S\&P Capital IQ

can select or design, an alternative benchmark that can provide better baseline exposure in the context of their overall portfolio.

We first consider an EM benchmark that continues to group stocks by country exposures, as does a traditional EM benchmark, but rather than using market-capitalization weights, the alternative benchmark uses either ERC or EW for the benchmark's construction. We call these "countrybased alternative" benchmarks to distinguish them from a traditional EM benchmark.

As a second benchmark alternative, we group EM stocks first by sector exposure, irrespective of country. For example, an Indian technology stock and a Brazilian technology stock would be assigned to the same EM technology sector (using market-capitalization weights). Then, to construct the alternative benchmark, we again combine the various sectors 


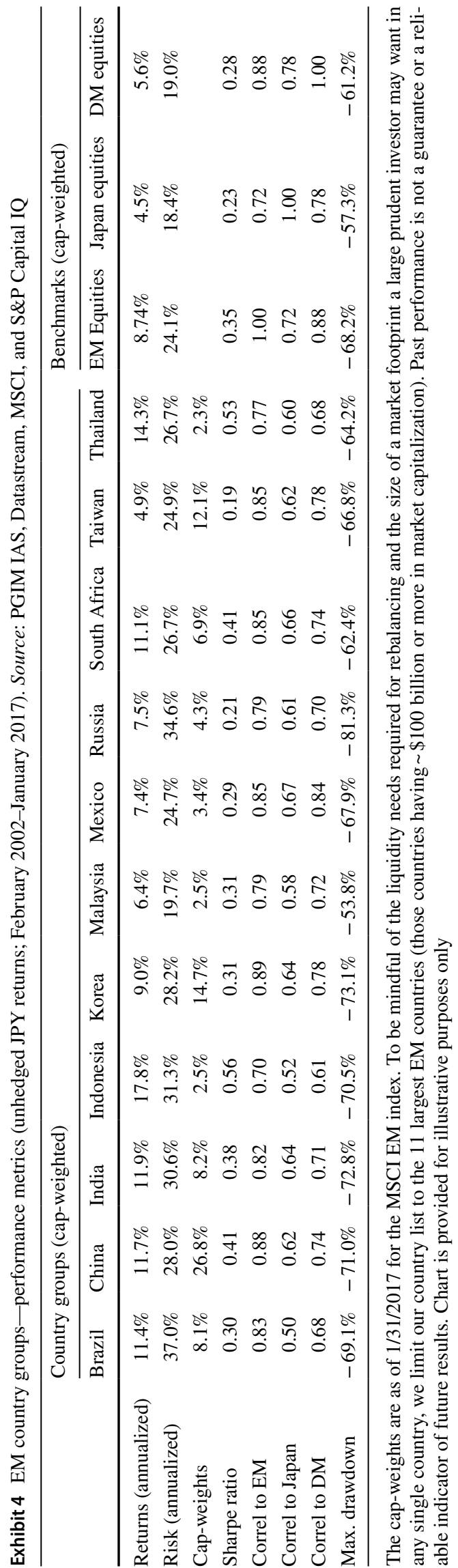

using ERC or EW. We call these "sector-based alternative" benchmarks. ${ }^{12}$

As a third alternative, we take advantage of academic research that has identified style exposures (e.g., momentum and value) as drivers of equity returns. Research suggests economic or behavioral motivations that allow stocks with these style exposures to generate positive, risk-adjusted returns. ${ }^{13}$ To construct "style-based alternative" benchmarks, we first classify stocks from a country into style groups depending on their exposures to these styles (using market-capitalization weights). ${ }^{14}$ Then, we combine these style groups using ERC or EW. As we show, for Japanese investors a style-based alternative EM benchmark has provided not only better returns, but also lower risk compared to a traditional EM benchmark.

For a given exposure grouping method, we construct an alternative benchmark using either ERC or EW. Both construction methodologies follow a set of rules. ERC (also known as "risk parity") is not new and has been well studied. ${ }^{15}$ ERC produces a risk-balanced benchmark such that each group's contribution to the benchmark's overall risk (volatility) is same for all groups in the benchmark. An investor considering an EM allocation may wish to adopt this approach given the higher risk involved with EM equities. Another benefit of ERC is that it does not rely on expected return assumptions. To construct a benchmark using ERC, we need only the volatilities and correlations of the various EM groups which we estimate each month using a widening window of historical data. This generates comparative results that are out-of-sample, providing an unbiased indication of how the various benchmark alternatives might perform going forward. Since the parameters are estimated each month, there is a potential for high turnover as alternative benchmarks are rebalanced. However, as shown below, by using a widening window of data, the degree of turnover is low.

The EW portfolio construction approach provides naïve diversification and does not require estimating any expected return or risk parameters. However, the portfolio has higher

\footnotetext{
${ }^{12}$ Investor may also embark on fundamental bottom-up thematic investing in EM, in new themes like "Modernizing infrastructure," "EM-EM cross-border trade," "New domestic consumer class," or "Fintech, e-commerce and distributional logistics." Such themes can also be tied to sector-based equity investing. See "Emerging Markets at the Crossroads," PGIM, June 2017.

${ }^{13}$ Refer to Fama and French (2016).

${ }^{14}$ Factors are generally interpreted as dollar-neutral long and short portfolios. Since we use long-only construction, to avoid any confusion, we refer to our approach as "style-based" benchmarking.

15 Refer to Maillard et al. (2010).
} 
risk and since the EM universe is generally very volatile, it may have lower value for Japanese investors.

\section{"Country-based alternative" benchmark approach}

Exhibit 4 shows that the EM country universe is not homogeneous. There is significant performance dispersion. From February 2002 to January 2017, there was almost a 13-percentage point range in total returns (in JPY) across countries. For example, while Indonesia had almost double the performance of the overall EM market (17.8 vs. 8.7\%), Taiwan performed meaningfully worse $(4.9 \%)$. There was also wide variation in EM country risk (as measured by annualized standard deviation of monthly returns): Brazil had 50\% greater risk compared to the overall EM market (37.0 vs. $24.1 \%$ ) while Malaysia had 20\% lower risk (19.7\%). Most notably, the three largest EM countries had among the highest correlations to Japan and DM ex-Japan. Countries with relatively low weights in the MSCI EM index (e.g., Indonesia, Malaysia and Thailand) had lower correlations to Japan and DM ex-Japan.

These country-level performance metrics suggest the possibility of constructing a better diversified EM benchmark for Japanese investors by reducing the weights of the larger EM countries and increasing those of the smaller countries. However, moving away from market-capitalization weights raises the issue of investability. While some EM countries have free float adjusted market caps that compare favorably with mid-cap sizes in single name US stocks, other EM countries are much smaller. Accordingly, we limit the EM country list to the 11 largest countries (approximately $\$ 100$ billion in market capitalization).

We weight these 11 countries to form an ERC "countrybased alternative" EM benchmark. Unlike for a market-capweighted index, due to relative market movements in EM country groups, rebalancing of the benchmark back to the target ERC weights at the end of each month is required. The monthly rebalancing causes the average annual two-way turnover to be $75 \%{ }^{16}$

Unlike a cap-weighted index, we do not observe significant concentration in handful of countries.

The equally weighted (EW) "country-based alternative" EM equity benchmark is constructed by allocating equally to all 11 countries. Weights are also rebalanced back to equal weights at each month end.

\footnotetext{
${ }^{16}$ At portfolio level, 75\% annual turnover in EM country groups is negligible (for e.g., at 5\% weight in EM equities of total portfolio, the annual turnover would be $3.75 \%$ ).
}

\section{"Sector-based alternative" benchmark approach}

We use GICS (Global Industry Classification Standard) to first group all EM stocks into 11 sectors (using marketcapitalization weights). ${ }^{17}$ As with country exposures, there is similar concentration among EM stocks in terms of their sector exposures. Almost $50 \%$ of EM stocks, by capitalization, belong to the globally oriented Finance and IT sectors (Exhibit 5). In contrast, the more defensive and arguably, more "local" sectors (e.g., Healthcare, Utilities, Telecom and Consumer-Staples) — which have lower correlation with DM ex-Japan-are less than 20\%. For investors seeking to benefit from faster internal growth in emerging markets, a weighting scheme tilted toward the smaller, more local sectors might be advantageous. For February 2002-January 2017, while not as large a range as with EM country groups, we find a returns range, across EM sectors, of 7.4 percentage points.

Due to relative market movements in EM sector groups, there is a need for monthly rebalancing of benchmark back to ERC target weights. The monthly rebalancing causes average annual two-way turnover to be $66 \%$.

ERC addresses the sector concentration issue. Instead of more than a $24.2 \%$ weight to financials, the weight in sectorbased alternative benchmark is only $8.1 \%$ (as of $1 / 31 / 2017$ ). As the volatility and correlations of healthcare sector were lower compared with the other sectors, it had the highest weight in the sector-based alternative benchmark (refer to online supplement A2). To put this in context, most recently, the healthcare sector had a $13.1 \%$ weight in the sector-based alternative benchmark but only a $2.4 \%$ weight in the traditional EM index.

The equally weighted (EW) "sector-based alternative" EM equity benchmark is constructed by allocating equally to all 11 sectors. Weights are rebalanced back to equal weights at each month end.

\section{"Style-based alternative" benchmark approach}

Finally, we consider alternative EM benchmarks based on style exposures. Market fundamentals may vary considerably across EM countries. Anecdotally, Indian equities have typically traded at relatively higher P/E multiples compared to

\footnotetext{
${ }^{17}$ In 2016, real estate was separated from the financial sector to form the 11th separate sector as per GICS (Global Industry Classification Standard). As the individual sectors are more than $\$ 100$ billion (as of $1 / 31 / 2017$ ), we do not attempt to construct sector groups using only stocks from the 11 largest countries we chose for country groups.
} 
other EM countries like China or Russia. This does not necessarily imply that the Indian equity market is too expensive and should be avoided. Relative to its historical average it may, in fact, be trading at a relatively low multiple and therefore may be of value. Therefore, we construct style groups first at the country level. To do so, we sort the stocks for each country based on a specific style (e.g., book-to-price), and the top quintile stocks within a country are combined (using market-capitalization weights) to form a country-level style group (e.g., the Brazil book-to-price style group). ${ }^{18}$ Similarly, for size and low-vol country-level style groups, the top quintile stocks in a given country represent stocks with the lowest volatility and the lowest $\log$ (market capitalization), respectively. Each country-level style group is rebalanced every month using prior month-end data. Then, for each of the seven styles considered here, we combine the 11 country-level style groups using ERC to produce an ERC EM style group. For example, the ERC EM book-to-price value style group is defined as the ERC combination of all 11 country-level book-to-price value style groups.

Exhibit 6 shows performance metrics for the seven longonly EM style groups ("value"-book-to-price and earnings-to-price ${ }^{19}$; "momentum"-12-month less one-month price returns; "carry"- dividend-to-price; "profitability"return-on-equity; "low volatility"-12-month volatility; and "size"- $\log ($ market capitalization $)) .{ }^{20}$

The range in returns among the seven ERC EM style groups was 8.0 percentage points, like the EM sector groups. We also observe that the EM size style group underperformed the traditional EM index (7.2 vs. 8.7\%). This is contrary to academic findings for DM large cap equities. One might argue that the analysis period of 15 years is too short,

\footnotetext{
18 Source: S\&P Capital IQ; The country level style portfolio constituents are from S\&P Broad Market Index (BMI) universe. For instance, Brazil Momentum, long-only style factor is the top quintile portfolio with stocks (from S\&P BMI universe and domiciled in Brazil) sorted based on 12 month-1 month momentum. The constituents are then market capitalization-weighted. In S\&P Capital IQ, the stock data coverage significantly improved from 2002 and therefore, we restricted our analysis from February 2002 to January 2017. For example, while in January 2001, Brazil stock coverage was $77 \%$, in January 2002, coverage improved to $98 \%$.

${ }^{19}$ We include both book-to-price and earnings-to-price value styles as their factor correlations are only 0.33 , which suggests these are distinct value styles in EM.

${ }^{20}$ We chose these seven styles due to their robust performance in the US markets, as demonstrated by the academic literature. US investors have also widely invested in these styles. We now verify their efficacy in an out-of-sample universe-EM equities. Refer to online supplement A3 for style definitions.
} 
Exhibit 6 EM style groups-performance metrics (unhedged JPY returns; February 2002-January 2017). Source: PGIM IAS, Datastream, MSCI, and S\&P Capital IQ

\begin{tabular}{|c|c|c|c|c|c|c|c|c|c|c|}
\hline & \multicolumn{7}{|c|}{ Style groups (equal risk contribution) } & \multicolumn{3}{|c|}{ Benchmarks (cap-weighted) } \\
\hline & MOM & $\mathrm{B} / \mathrm{P}$ & $\mathrm{E} / \mathrm{P}$ & Size & Low-vol & $\mathrm{D} / \mathrm{P}$ & ROE & EM equities & Japan equities & DM equities \\
\hline Returns (annualized) & $15.2 \%$ & $11.2 \%$ & $14.0 \%$ & $7.2 \%$ & $9.9 \%$ & $13.0 \%$ & $10.7 \%$ & $8.7 \%$ & $4.5 \%$ & $5.6 \%$ \\
\hline Risk (annualized) & $23.8 \%$ & $27.0 \%$ & $25.5 \%$ & $25.9 \%$ & $19.5 \%$ & $23.1 \%$ & $22.5 \%$ & $24.1 \%$ & $18.4 \%$ & $19.0 \%$ \\
\hline Sharpe ratio & 0.63 & 0.41 & 0.54 & 0.27 & 0.50 & 0.56 & 0.47 & 0.35 & 0.23 & 0.28 \\
\hline Correl to EM & 0.95 & 0.95 & 0.96 & 0.94 & 0.95 & 0.95 & 0.97 & 1.00 & 0.72 & 0.88 \\
\hline Correl to Japan & 0.71 & 0.69 & 0.71 & 0.72 & 0.75 & 0.72 & 0.74 & 0.72 & 1.00 & 0.78 \\
\hline Correl to DM & 0.83 & 0.82 & 0.85 & 0.84 & 0.87 & 0.83 & 0.86 & 0.88 & 0.78 & 1.00 \\
\hline Max. drawdown & $-70.6 \%$ & $-69.1 \%$ & $-67.6 \%$ & $-73.3 \%$ & $-61.7 \%$ & $-62.0 \%$ & $-70.5 \%$ & $-68.2 \%$ & $-57.3 \%$ & $-61.2 \%$ \\
\hline
\end{tabular}

Past performance is not a guarantee or a reliable indicator of future results. Chart is provided for illustrative purposes only

Exhibit 7 Equal risk

contribution weighted EM benchmarks-performance metrics (unhedged JPY returns; February 2002-January 2017). Source: PGIM IAS, Datastream, MSCI, and S\&P Capital IQ

\begin{tabular}{lccccccc}
\hline & \multicolumn{2}{l}{ Benchmarks (equal risk contribution) } & & \multicolumn{2}{c}{ Benchmarks (cap-weighted) } \\
\cline { 2 - 3 } & EM countries & EM sectors & EM styles & & EM equities & Japan equities & DM equities \\
\hline Returns (annualized) & $11.6 \%$ & $9.7 \%$ & $11.4 \%$ & & $8.7 \%$ & $4.5 \%$ & $5.6 \%$ \\
Risk (annualized) & $23.3 \%$ & $22.4 \%$ & $22.8 \%$ & & $24.1 \%$ & $18.4 \%$ & $19.0 \%$ \\
Sharpe ratio (SR) & 0.49 & 0.42 & 0.49 & & 0.35 & 0.23 & 0.28 \\
$p$ Value (SR diff) & 0.00 & 0.08 & 0.07 & & & \\
Correl to EM & 0.99 & 0.99 & 0.98 & & 1.00 & 0.72 & 0.88 \\
Max. drawdown & $-66.1 \%$ & $-64.2 \%$ & $-66.1 \%$ & & $-68.2 \%$ & $-57.3 \%$ & $-61.2 \%$ \\
\hline
\end{tabular}

We ignore transaction costs from monthly rebalancing back to ERC weights for the three alternative EM benchmarks (we approximate cost to be $33 \mathrm{bp}$ (avg. transaction cost) $\times 75 \%$ (average annual two-way turnover), adds to $25 \mathrm{bp} / \mathrm{y}$ ). However, performance metrics are reported after deducting estimated transaction cost for country-level style groups, which unlike country or sector market-cap-weighted groups, have higher turnover (refer to online supplement A4). We estimate transaction costs using average turnover, bidask spreads and taxes. We estimated the cost to be $1 \% / y$ for EM styles. Past performance is not a guarantee or a reliable indicator of future results. Chart is provided for illustrative purposes only as these robust sources of risk premia can go in and out of favor for more than a decade. Nevertheless, we exclude the EM size style group from the construction of the style-based alternative EM benchmark. ${ }^{21}$

Finally, we combine the six EM style groups, using either ERC or EW, to form "style-based alternative" EM benchmarks. Due to relative market movement in EM style groups, the benchmark needs to be rebalanced back to ERC target weights on a monthly basis. The monthly rebalancing causes the two-way average annual turnover to be $62 \%$.

To construct the equally weighted (EW) "style-based alternative" EM equity benchmark, we equally weight the six ERC EM style groups. The benchmark is rebalanced at each month end.

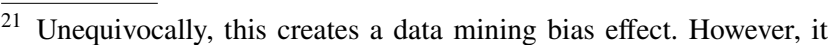
does not make sense to include size if it does not work in majority of the countries. We believe "investable," "governance" and "crosslisting" premia can be masking size effect. Refer to O'Connor et al. (2014).
}

\section{Performance comparison of EM benchmarks}

Exhibit 7 shows that the returns for all three alternative benchmarks using ERC were highly correlated with the traditional EM index, suggesting that they all adequately captured exposure to the EM market. ${ }^{22,23}$ However, all three alternative benchmarks had better absolute and risk-adjusted

\footnotetext{
$\overline{22}$ Using EW, EM countries returned $11.8 \%$ with risk of $23.5 \%$, EM sectors returned $9.4 \%$ with risk of $22.9 \%$ and EM Styles returned $11.7 \%$ with risk of $23.3 \%$. EM countries, sectors and styles correlations to EM cap-weighted index were $0.99,1.0$ and 0.97 , respectively. If size style was included, EM styles returned $10.9 \%$ with risk of $23.4 \%$ and correlation to cap-weighted EM index of 0.97.

${ }^{23}$ What would the performance have been say for the US investor who would have invested in the three alternative EM benchmarks? In USD currency, using EW, EM countries returned $13.1 \%$ with risk of $21.6 \%$, EM sectors returned $10.7 \%$ with risk of $20.7 \%$ and EM styles returned $13.0 \%$ with risk of $16.2 \%$. EM countries, sectors and styles correlations to EM cap-weighted index were $0.99,0.99$ and 0.95 , respectively. In comparison, EM cap-weighted index returned $10.0 \%$ with risk of $22.0 \%$.
} 

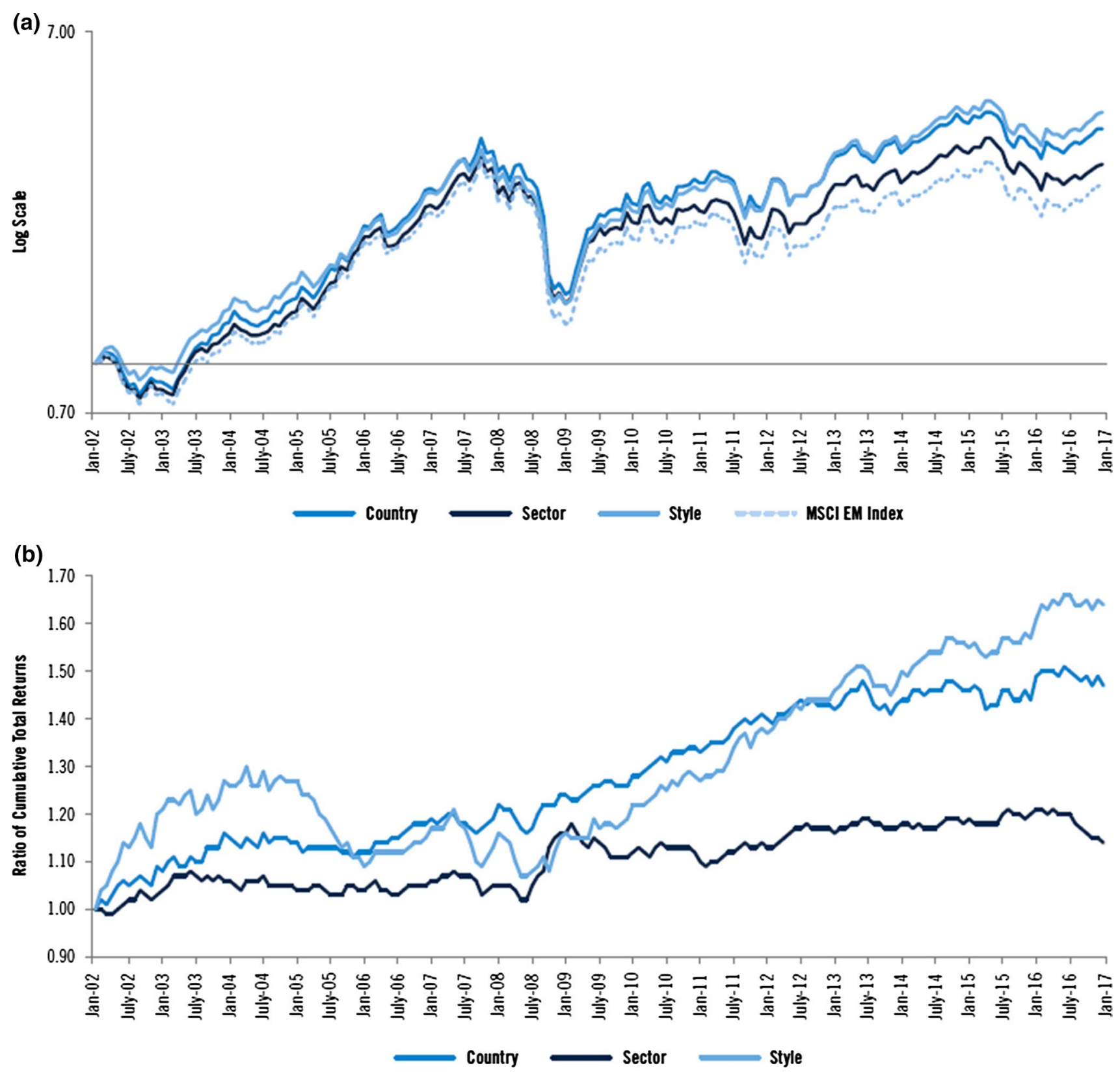

Exhibit 8 a Cumulative total returns (unhedged JPY returns; February 2002-January 2017). b Cumulative total returns ratio alternative benchmarks relative to MSCI EM index (unhedged JPY returns; February 2002-January 2017). Note: Past performance is not a guarantee

performance compared to the traditional EM benchmark. ${ }^{24}$ In addition, all three alternative benchmarks had lower

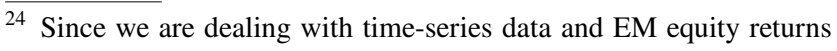
are non-normal, we use HAC (heteroskedasticity and autocorrelation robust kernel estimation) inference to determine if Sharpe ratios of the three proposed benchmarks are statistically different enough from that for the market capitalization-weighted EM benchmark based on the $\mathrm{p}$ values calculated. Refer to Ledoit and Wolf (2008). Lower $p$ value suggests the two Sharpe ratios are different from each other.
}

or a reliable indicator of future results. Chart is provided for illustrative purposes only. Source: PGIM IAS, Datastream, MSCI, and S\&P Capital IQ

absolute return maximum drawdowns, reflecting the contribution of ERC in helping to control risk. The sector-based alternative benchmark had the lowest risk and maximum drawdown but also the lowest return. 
Exhibit 9 Benchmark performance in various economic scenarios (unhedged JPY returns; February 2002-January 2017). Source: PGIM IAS, Datastream, MSCI, and S\&P Capital IQ

\begin{tabular}{|c|c|c|c|c|c|c|}
\hline & \multicolumn{3}{|c|}{ Benchmarks (equal risk contribution) } & \multicolumn{3}{|c|}{ Benchmarks (cap-weighted) } \\
\hline & EM countries $(\%)$ & EM sectors $(\%)$ & EM styles (\%) & EM equities (\%) & Japan equities (\%) & $\begin{array}{l}\text { DM ex-Japan } \\
\text { equities (\%) }\end{array}$ \\
\hline Weak yen & 20.7 & 19.7 & 21.3 & 18.2 & 20.3 & 18.0 \\
\hline Strong yen & 5.2 & 2.8 & 6.1 & 2.2 & -6.0 & -2.7 \\
\hline High uncertainty & 4.5 & 2.9 & 4.2 & 1.5 & -7.3 & -1.8 \\
\hline Low uncertainty & 17.8 & 15.7 & 19.7 & 15.1 & 15.5 & 12.4 \\
\hline Weak oil & 6.6 & 6.3 & 7.9 & 3.5 & 5.4 & 3.1 \\
\hline Strong oil & 14.4 & 11.6 & 15.0 & 11.8 & 4.0 & 7.2 \\
\hline US recession & -14.9 & -16.1 & -12.1 & -19.2 & -10.3 & -15.9 \\
\hline US expansion & 26.5 & 24.2 & 25.9 & 24.8 & 12.1 & 17.5 \\
\hline JP recession & -6.5 & -6.1 & -5.2 & -8.3 & -4.0 & 0.4 \\
\hline JP expansion & 26.2 & 22.3 & 26.5 & 22.4 & 10.9 & 9.6 \\
\hline Weak EM flows & -11.8 & -12.7 & -10.3 & -14.8 & -6.6 & -9.8 \\
\hline Strong EM flows & 36.4 & 33.4 & 36.2 & 34.0 & 15.0 & 21.1 \\
\hline
\end{tabular}

Determination of "strong" or "weak" is based on if the value is above or below its 12-month moving average. In case of JPY/USD if the currency is above 12 month moving average, we label the months as "Weak Yen." We use the World Economic Policy Uncertainty index from www.economicpolicyuncertainty.com. US and Japan recession are sourced from FRED (Federal Reserve Bank of St. Louis) based on OECD composite leading indicators following peak-to-trough marked as recession. The strong EM flows represent higher annual flows in EM than previous year (Source: IIF). Past performance is not a guarantee or a reliable indicator of future results. Chart is provided for illustrative purposes only

\section{Long-term performance comparison of alternative EM benchmarks}

All three alternative ERC EM benchmarks outperformed the traditional EM index (Exhibit 8a). The style-based alternative benchmark has particularly outperformed the traditional EM benchmark since 2012 (Exhibit 8b). As shown, the sector-based alternative benchmark has not performed as well as the country-based or style-based alternative benchmarks.

The sector-based benchmark eked out most of its cumulative relative outperformance versus the MSCI EM index during the financial crisis. While the alternative benchmark's relative overweight to healthcare and consumer-staples helped, its relative overweight to real estate hurt, as the sector had the highest volatility and the largest maximum drawdown of all sectors. The longest period the sector-based benchmark underperformed relative to the traditional EM index was the five-year period July 2003 to August 2008.

During the strong EM rally beginning in 2005, all the styles (except momentum) did not keep up with the overall market index. The style-based benchmark underperformed relative to the MSCI EM index for over 7 years from May 2004 to May 2011. From the October 2008 relative underperformance trough, it took almost 3 years for the alternative style benchmark to regain its relative advantage. Clearly, the style-based approach should be adopted by investors with a longer investment horizon.
Exhibit 10 Systematic market exposures (February 2002-January 2017). Source: PGIM IAS, Datastream, MSCI, and S\&P Capital IQ

\begin{tabular}{lllll}
\hline & \multicolumn{4}{l}{ Relative to 50\% DM-ex JP and 50\% JP equities } \\
\cline { 2 - 5 } & EM countries & EM sectors & EM styles & EM equities \\
\hline Beta & 1.12 & 1.09 & 1.10 & 1.16 \\
Alpha & $6.2 \%$ & $4.5 \%$ & $6.0 \%$ & $3.5 \%$ \\
R-squared & 0.74 & 0.74 & 0.73 & 0.74 \\
\hline
\end{tabular}

EM country, sector and style benchmarks are ERC weighted, and EM equities are market-capitalization weighted. The transaction costs are estimated using average turnover, bid-ask spreads and taxes (refer to online supplement A4). We estimate transaction costs to be $1 \% / y$ for EM styles. Chart is provided for illustrative purposes only

On relative basis, the country-based alternative benchmark steadily outperformed the MSCI EM index and its longest period of relative underperformance was for only 2 years, August 2004 to July 2006.

Overall, the country-based and style-based alternative benchmarks, based on their long-term performance, are worthy of consideration by Japanese investors.

\section{Alternative benchmark performance in various economic scenarios}

We next evaluate the performance of the three alternative EM benchmarks in a handful of economic scenarios likely to be of interest to a Japanese investor. The scenarios include: 
Exhibit 11 Sample Japanese portfolio allocation for a Japanese investor-performance metrics EM equities at 5\% allocation (JPY returns; February 2002-January 2017). Source: PGIM IAS, Datastream, MSCI, and S\&P Capital IQ

\begin{tabular}{lllllc}
\hline & Plan & Plan with EM equity & Plan with EM countries & $\begin{array}{l}\text { Plan with EM sectors } \\
\text { Plan with } \\
\text { EM styles }\end{array}$ \\
\hline Returns (annualized) & $4.50 \%$ & $4.69 \%$ & $4.81 \%$ & $4.72 \%$ & $4.80 \%$ \\
Risk (annualized) & $9.59 \%$ & $9.71 \%$ & $9.68 \%$ & $9.65 \%$ & $9.66 \%$ \\
Sharpe ratio (SR) & 0.45 & 0.46 & 0.48 & 0.47 & 0.48 \\
$p$ Value (SR diff) & & 0.45 & 0.14 & 0.26 & 0.17 \\
\hline
\end{tabular}

Country, sector and style EM benchmarks are ERC weighted. We ignore the transaction costs from monthly rebalancing of the benchmarks to ERC weights. However, performance metrics are reported after deducting estimated transaction costs for country-level style groups, which unlike country or sector market-cap-weighted groups, have higher turnover (refer to online supplement A4). The transaction costs are estimated using average turnover, bid-ask spreads and taxes. We estimated the cost to be $1 \% / y$ for EM Styles. Past performance is not a guarantee or a reliable indicator of future results. Chart is provided for illustrative purposes only

macro events like recession and expansions; market events such as commodity and currency price movements; the strength of money flows into EM equity markets; changes in economic uncertainty as implied by world news.

Exhibit 9 shows that EM equities were an excellent diversifier to DM ex-Japan and Japan equities in some scenarios such as strong yen or high economic uncertainty. In the US and Japan recession scenarios-which can be categorized as "risk off" events, the style-based alternative benchmark did significantly better than other EM benchmarks. In fact, for the US recession scenario, country-based and style-based benchmarks also outperformed DM ex-Japan equities. In more "risk on" scenarios, both country-based and stylebased benchmarks also did better. This analysis supports consideration of country-, sector- or style-based alternative benchmarks by Japanese investors.

\section{Systematic risk in the alternative benchmarks}

We have showed the risk-adjusted performance of these three alternative EM benchmarks, but what kind of systematic risk do they bear? We can measure that by looking at the systematic exposure (beta) of these benchmarks to the equity exposure currently held by many Japanese institutional investors.

To determine which alternative benchmark had the lowest systematic exposure to a Japanese investor's current equity allocation, we regressed each EM benchmark against a 50\% DM ex-Japan-50\% Japan market-capitalization-weighted equity index. The regression results (Exhibit 10) show reduced exposure (beta) to the market for the three alternative EM benchmarks than for the traditional EM index. The sector-based benchmark had the lowest beta to the market. The annualized regression alpha for the MSCI EM index was $3.5 \%$, but the three alternative benchmarks had even higher alphas. Country-based and style-based benchmarks have comparable alphas $(6.2 \%$ and $6.0 \%$, respectively).

\section{Alternative EM benchmark in Japanese investor portfolios}

To keep the allocation to yen and equities unchanged, we assume EM allocation is made by reducing the allocation to DM ex-Japan from the sample Japanese portfolio. To assess relative risk-adjusted returns, we allocate $5 \%$ from DM ex-Japan equities to EM equities (i.e., $10 \%$ of the overall equity allocation). We also incorporate transactions costs. ${ }^{25}$ We find that using any of the four EM benchmarks (i.e., the three alternative ERC benchmarks and the traditional EM benchmark), both risk and return increased (Exhibit 11). The best performance improvement came from using the country-based alternative benchmark (an improvement of $31 \mathrm{bp}, 4.81$ vs. $4.50 \%)$.

\begin{abstract}
25 At the portfolio level, we ignore the transaction costs from monthly rebalancing of the alternative benchmarks back to ERC weights as it is negligible. For example, for the country-based ERC alternative benchmark, 33 bp (avg. transaction cost) $\times 5 \%$ (portfolio weight) $\times 75 \%$ (average annual two-way turnover), adds to $1 \mathrm{bp} / \mathrm{y}$ at the overall portfolio level. Besides, the country and sector groups used to construct the country-based and sector-based alternative EM benchmark are market capitalization-weighted and therefore they incur negligible transaction costs as well. However, this is not the case for the underlying country-level style groups used to form a EM style group. From month-to-month, there can be changes in the set of stocks forming the top quintile of a country's style group, which necessitates turnover. Refer to online supplement A4, where we estimate turnover for each of the country-level style groups. Due to high turnover in the country-level style groups, we estimate the annual two-way transaction cost to be $1 \% / y$ for the EM style-based benchmark; at the portfolio level, and this adds up to $5 \mathrm{bp} / \mathrm{y}$ for a $5 \% \mathrm{EM}$ allocation. While a benchmark rarely includes transaction costs, a portfolio that tracks a benchmark does incur this cost. To be conservative, we assume that a portfolio that adopts a style-based alternative benchmark should account for the likely higher transactions costs. Consequently, to facilitate comparison across the various benchmarks, we report returns for the style-based alternative benchmark after accounting for likely transaction costs.
\end{abstract}


We conducted difference in means test to compare the two Sharpe ratios, one for the portfolio with no EM equity allocation and the other for the portfolio with one of the four EM benchmark equity allocations. A lower $p$ value for the test would suggest that the Sharpe ratios are indeed different, and the investor may wish to consider the allocation choice with the highest Sharpe ratio. Exhibit 11 indicates that a Japanese investor may wish to consider either the country- or style- alternative EM benchmarks as superior alternatives. For EW alternative benchmarks, the risk increases slightly without any improvement in the Sharpe ratios (not shown).

To summarize, the three alternative benchmarks (using ERC), after accounting for transaction costs, perform better than the traditional EM index from the Japanese investor's perspective. Not only did the three alternative benchmarks have superior returns when compared with the traditional EM index, but all three improved the sample Japanese portfolio's Sharpe ratio, thereby justifying the consideration of adopting an alternative EM benchmark.

\section{Conclusion}

Japanese investors maybe considering adding EM equities to their portfolios. How might a Japanese investor select, or design, an EM benchmark? We have shown that a traditional market-capitalization-weighted EM index has unwanted risk concentrations, both at country-level and sector-level, which may not be desirable for the Japanese investor. We propose "country-based," "sector-based" or "style-based" alternative EM benchmarks which may be better-suited to a Japanese investor's circumstances. When constructing these benchmarks, we also use alternative weighting schemes-ERC and equal-weight. There are many other potential exposure weighting schemes, and some may work better than others depending on the economic regime.

For Japanese investors, adding EM equities in lieu of DM ex-Japan equities increases portfolio risk, but it came with a twofold benefit: portfolio diversification and performance enhancement. Historically, Japanese investors would have found limited improvement in their overall portfolio's riskadjusted returns using a traditional market-capitalization EM benchmark to serve as their baseline allocation. Instead, Japanese investors would have done better using an EM alternative benchmark.

All the three alternative benchmarks had a significantly higher Sharpe ratio than the traditional EM index. While all three alternative benchmarks had better performance than the traditional EM index, the country-based and style-based alternative benchmarks performed better than the sectorbased alternative benchmark. With the trend of improved EM equity data and coverage and reduction of transaction costs, Japanese investors may wish to consider adopting an alternative EM benchmark for their new EM allocation.

\section{End notes}

This article is for educational purposes only and is not intended to be used as an investment advice or recommendation by person or persons in possession of this material. For the Appendix, complete disclosures, and important information, please refer to the online supplement.

Open Access This article is distributed under the terms of the Creative Commons Attribution 4.0 International License (http://creativeco mmons.org/licenses/by/4.0/), which permits unrestricted use, distribution, and reproduction in any medium, provided you give appropriate credit to the original author(s) and the source, provide a link to the Creative Commons license, and indicate if changes were made.

\section{References}

Amenc, N., F. Goltz, A. Lodh, and L. Martellini. 2012. Diversifying the Diversifiers and Tracking the Tracking Error: Outperforming Cap-Weighted Indices with Limited Risk of Underperformance. Journal of Portfolio Management 38 (3): 72-88.

Fama, E., and K. French. 2016. Dissecting Anomalies with a FiveFactor Model. Review of Financial Studies 29: 69-103.

Ledoit, O., and M. Wolf. 2008. Robust Performance Hypothesis Testing with Sharpe Ratios. Journal of Empirical Finance 15: 850-859.

Maillard, S., T. Roncalli, and J. Teiletche. 2010. The Properties of Equally Weighted Risk Contribution Portfolios. Journal of Portfolio Management 36: 60-70.

O’Connor, T., S. Kinsella, and V. O'Sullivan. 2014. Legal Protection of Investors, Corporate Governance, and Investable Premia in Emerging Markets. International Review of Economics \& Finance 29: 426-439.

Wilcox, J. 1994. EAFE is for Wimps. Journal of Portfolio Management 20 (3): 68-75.

Publisher's Note Springer Nature remains neutral with regard to jurisdictional claims in published maps and institutional affiliations.

Harsh Parikh has extensive empirical research experience and has published in few peer-reviewed journals. He is a practitioner with over 15 years of financial services experience, most recently as a thought leader and an adviser to large institutional clients. 